\title{
Predictors of mortality in hospitalized patients with influenza: a five-year experience from a tertiary care centre in Pakistan
}

\author{
Iffat Khanum, Amber Sabeen Ahmed, Safia Awan, Sabiha Bano, Bushra Jamil \\ Department of Medicine, Aga Khan University Hospital, Karachi, Pakistan
}

\begin{abstract}
Influenza outbreaks are associated with significant morbidity. Our aim was to determine the factors associated with increased mortality in hospitalized patients admitted with diagnosis of influenza, at a tertiary care center in Pakistan. This study included all adult patients with an influenza infection, confirmed by realtime reverse-transcriptase polymerase-chain-reaction (RT-PCR) at Aga Khan University Hospital Pakistan. In our study, 112 patients with laboratory-confirmed influenza virus infection were admitted at our hospital from the $1^{\text {st }}$ of January 2013 to the $31^{\text {st }}$ of December 2018. Eighty-nine patients (79.46\%) were managed in ward or special care units and 23 patients $(20.5 \%)$ received treatment in intensive care unit (ICU). The overall mortality in our study was $15 / 112(13.4 \%)$ with the mortality rate of ICU patients
\end{abstract}

Correspondence: Amber Sabeen Ahmed, Department of Medicine, Aga khan University hospital, Stadium Road, Karachi, Pakistan. Tel. +92.2134864124.

E-mail: ambersabeen@hotmail.com

Key words: Influenza; mortality; predictors.

Author's contributions: All authors contributed equally.

Conflict of interest: The authors declare no conflict of interest.

Conference presentation: MMIDSP annual conference 2020; Accepted oral presentation Gulf Chest Conference (postponed due to COVID pandemic).

Funding: None.

Ethics approval: The study is approved by the Ethical Review Committee of Aga Khan University Hospital.

Informed consent: Not applicable.

Received for publication: 13 June 2020.

Accepted for publication: 3 August 2020.

${ }^{\circ}$ Copyright: the Author(s), 2020

Licensee PAGEPress, Italy

Monaldi Archives for Chest Disease 2020; 90:1456

doi: 10.4081/monaldi.2020.1456

This article is distributed under the terms of the Creative Commons Attribution Noncommercial License (by-nc 4.0) which permits any noncommercial use, distribution, and reproduction in any medium, provided the original author(s) and source are credited. being $47.8 \%$ while the mortality rate of patients treated in special care units and wards was only $4.5 \%$. The mean age of patients with influenza infection was 58.1 years $( \pm 16.6)$. Influenza virus type A was found in 87 patients (77.6\%), while influenza type B was present in only $25(22.4 \%)$ patients. Out of the 15 non-survivors, 14 had influenza A. Only 17 patients (15.2\%) were found to have positive culture of respiratory specimen, out of which 3 were non-survivors and 14 were survivors. Our analysis identified septic shock (odds ratio 45.24; 95\%, confidence interval 6.20-330; $\mathrm{p}<0.001$ ), renal failure (odds ratio $10.88 ; 95 \%$, confidence interval $1.61-73.52 ; \mathrm{p}=0.01$ ) and ICU stay (odds ratio 17.22 ; $95 \%$, confidence interval 2.68-110.5; $\mathrm{p}=0.003$ ) as independent risk factors associated with in-hospital mortality.

\section{Introduction}

Influenza, commonly known as flu is an infection caused by influenza virus which is an RNA virus belonging to Orthomyxoviridae family [1]. Most of the epidemic of influenza is usually caused by two types of viruses, influenza A and influenza B. Influenza A is further subcategorized into subtypes e.g., influenza $A(\mathrm{H} 1 \mathrm{~N} 1)$ viruses, influenza $\mathrm{A}(\mathrm{H} 3 \mathrm{~N} 2)$ viruses based on characteristics of two surface antigens, hemagglutinin (HA) and neuraminidase (NA) [2]. The spectrum of disease from influenza virus ranges from mild flu like illness to acute respiratory distress syndrome (ARDS). Annual epidemics caused by influenza virus are not only a major burden to health care resources, but also associated with high mortality rates [3]. Globally, 3-5 million cases of influenza are reported per annum and the estimated global mortality rate is between 291,243-645,832 per year [4]. During 20172018 influenza season the overall influenza related mortality in Europe was estimated to be 25.4 (95\% CI $25.0-25.8)$ per 100,000 population [5].

The burden of disease in Asia is much higher than Europe. Eleven thousand seven hundred thirty-four $(11,734)$ laboratoryconfirmed influenza cases were reported in 19 Asian countries between 2010-2017 [6]. In 2015, about more than 30,000 cases and 1731 deaths were reported in India alone [7]. In a laboratorybased influenza surveillance study conducted between 2007 and 2017 a total of 3475 influenza cases were detected in Pakistan. In 2017, influenza related death from Pakistan was reported to be 2.0 per $100,000[8,9]$. Despite the annual epidemics of influenza in Pakistan there have been very few studies done on the disease from our country. To be better prepared to handle influenza epidemics and to decrease the mortality rates associated with the disease, it is of paramount importance to know the predictors of mortality of influenza. Respiratory failure is the major cause of mortality in patient with influenza infection [10]. A study from India, 
during influenza outbreak of 2015 showed that the requirement of intubation was the only significant predictor of mortality related to the disease [11]. In a meta-analysis, which included 600,000 patients, risk factors for influenza associated mortality were age, atrial fibrillation and pneumonia [12].

To our knowledge, there is no published five-years study from Pakistan on predictors of influenza related mortality. The purpose of this study is to identify factors associated with mortality in hospitalized influenza over a period of five years at tertiary care hospital in Pakistan.

\section{Methodology}

We conducted a retrospective cross-sectional study from January 13-December 18, at Aga Khan University Hospital in Pakistan which is a 750 bedded JCIA accredited tertiary care hospital. We included patients aged 18 and above, of both sexes, who had symptoms of influenza like illness (temperature of $37.8^{\circ} \mathrm{C}$ or higher along with cough or sore throat) confirmed by Real-time Reverse-Transcriptase Polymerase Chain-reaction (RT-PCR). Nasal swabs were taken under aseptic precautions of all patients admitted with influenza like illness.

Clinical data was collected from review of patient's daily records. Radiological and laboratory data were obtained from electronic records. Data was collected regarding baseline demographics, symptoms at time of admission, oseltamivir prescription, antibiotics, any positive cultures (blood, sputum, and urine), hospital and Intensive Care Unit (ICU) length of stay. All the interventions undertaken like invasive and non-invasive ventilation, renal replacement therapy, use of vasopressors, and cases of septic shock were recorded. Data was also obtained for the oxygen requirements, development of ARDS and use of steroids. Data regarding development of complications and clinical outcome at discharge was also collected. Ethical review committee of Aga Khan Hospital had approved the study.

\section{Statistical analysis}

A descriptive analysis was done for demographic and clinical features. The results were presented as mean \pm SD for quantitative variables and numbers (percentage) for qualitative variables. Differences in proportions, between survivors and non-survivors, were assessed by using the Chi-square test or Fisher exact test where appropriate. To assess univariate associations between the outcomes and potential predictors, odds ratios (ORs) and their $95 \%$ confidence intervals (CIs) were computed by logistic regression analysis. All p-values were two sided and considered as statistically significant if $<0.05$. All significant factors on univariate analysis were considered for inclusion in the multivariable logistic model.

All analyses were conducted by using the SPSS software, release 16.0 .

\section{Results}

In our study, 112 patients with laboratory confirmed influenza virus infection were admitted at our hospital from $1^{\text {st }}$ January 2013 to $31^{\text {st }}$ December 2018. Eighty-nine patients (79.46\%) were man- aged in ward or special care units and 23 patients $(20.5 \%)$ received treatment in ICU. Although the overall mortality in our study was $13.4 \%$, the mortality rate of patients admitted in ICU with influenza was $47.8 \%$. The mean age of patients with influenza infection was 58.1 years $( \pm 16.6)$. The majority of patients $(42 \%$ of the total number) were between 60 and 75 years of age but the $40-60$ years age group was found to have the highest mortality rate $(23.33 \%)$. Even though $62.5 \%(70 / 112)$ of all patients were female, male patients had higher mortality $(19.02 \% \mathrm{v} / \mathrm{s} 10 \%)$. Fourteen patients were pregnant and all but one of them survived.

A total of 72 out of 112 patients $(64.2 \%)$ had at least one co morbid illness. Hypertension $(n=54,48.2 \%)$ and Diabetes $(n=51$, $45.5 \%$ ) were two most common comorbid conditions in our study population. Other co-morbid observed were, chronic lung diseases $(n=32,28.6 \%)$, heart disease $(n=18,16.1 \%)$ and renal impairment $(n=12,10.71 \%)$, while neurological diseases were present in only 4 patients (3.5\%) (Figure 1). Among all co-morbid conditions, heart diseases were the only one which were more prevalent in non-survivors $(4 / 15,26.7 \%)$ compared to survivors $(14 / 89,14.4 \%$; the results though were not statistically significant). The time between onset of symptoms and hospital admission ranged from 125 days, with a median of 5 days. It was $5.1 \pm 2.7$ days in survivors and $7 \pm 5.2$ days in non-survivors. Fever (93\%) and cough $(90 \%)$ were the most common symptoms, followed by shortness of breath (61\%). The clinical presentations were equally distributed among survivors and non-survivors. Only 20 patients $(17.9 \%)$ received invasive mechanical ventilation, out of which 10 died. Forty patients received non-invasive ventilation.

Influenza virus type A was found to be the most common strain of influenza in this setting. Eighty-seven patients (77.6\%) were positive for influenza type A, while influenza type B was present in only $25(22.4 \%)$ patients. Out of the 15 non-survivors, 14 had influenza A.

Only 17 patients $(15.2 \%)$ were found to have positive culture of respiratory specimen, out of which 3 were non-survivors and 14 were survivors. Staphylococcus aureus was the most common isolated organism ( $37 \%$ of overall culture positive organism), followed by Aspergillus spp. (29\% of overall culture positive organism). Among non-survivors, two patients had sputum culture positive for Acinetobacter and 1 had positive sputum culture for $H$. influenzae. All patients received oseltamivir and the mean duration between the onset of symptoms and the initiation of therapy was

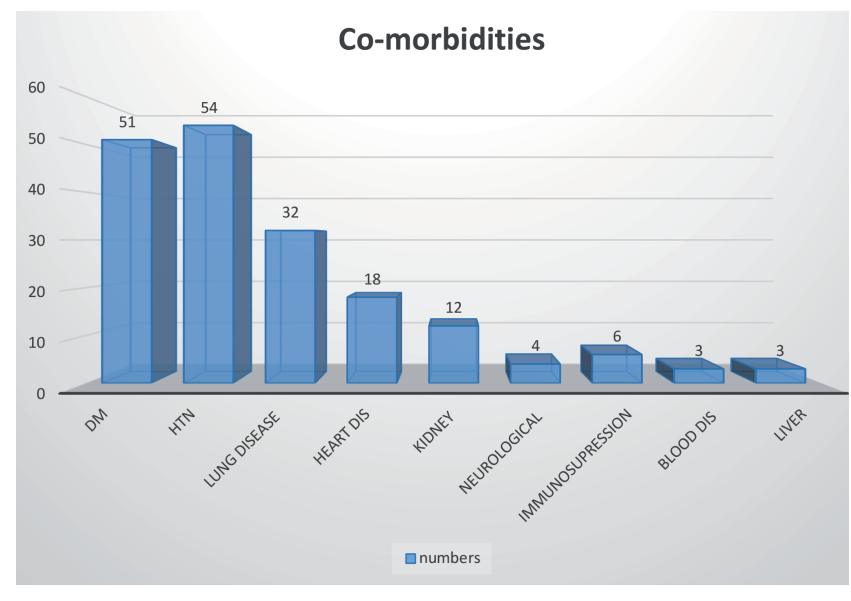

Figure 1. Influenza and comorbid illnesses. 
$5.3 \pm 3.3$ days. Delay in the initiation of oseltamivir was associated with increased complications.

The most common influenza related complications that were observed in our study was secondary bacterial pneumonia $(65 \%)$ followed by acute renal failure (30\%). Among survivors, renal failure was present in 24/89 (26.96\%) patients and among non-survivors, it was present in $10 / 15(66.66 \%)$ patients. ARDS was reported in 16/112 (14\%) of the patients, out of which 9 were nonsurvivors. Seventeen patients (15\%, of total influenza patients) were diagnosed with septic shock and it was found to be more prevalent in non-survivors. Eleven out of fifteen (73.33\%) nonsurvivors were found to have septic shock, while amongst survivors, only 6/89 (6.74\%) had the complication. Cardiac complications, as a group were present in 27 patients (24.1\%) among which 16 patients had acute coronary syndrome; among these patients 12 were survivors and 4 were non-survivors. Eleven patients developed arrhythmias during their hospital stay and only one of them was a non-survivor. Steroids were given to 61 patients $(54.5 \%)$, out of which, 10 of them were non-survivors $(66.66 \%)$. The average length of a hospital stay for a non-survivor was $12.23 \pm 7.8$ days, while that of survivor was $6.53 \pm 6.1$ days. The length of ICU stay was also longer for non-survivors as compared to survivors (10.23 \pm 6.6 days vs $7.73 \pm 5.8$ days).

Univariate and multivariate logistic regression was used to identify predictors of mortality in influenza (Table 1). The length of hospital stays ( $p=0.01$ ), prolonged duration between the onset of illness and hospital admission $(p=0.03)$, the length of ICU stays $(p<0.001)$, invasive ventilation $(p<0.001)$, ARDS $(p=0.001)$, respiratory failure $(\mathrm{p}=0.008)$, septic shock $(\mathrm{p}<0.001)$ and acute renal failure $(p<0.001)$ were considered to be significant in the univariate analysis. The multivariate analysis identified septic shock (odds ratio 45.24; 95\%. confidence interval 6.20-330; $<<0.001$ ), renal failure (odds ratio $10.88 ; 95 \%$. confidence interval 1.61 73.52; $\mathrm{p}=0.01$ ) and ICU stay (odds ratio $17.22 ; 95 \%$. confidence interval 2.68-110.5; $\mathrm{p}=0.003$ ) as independent risk factors associated with in-hospital mortality (Table 2).

\section{Discussion}

This study reported on a series of hospitalized patients with laboratory confirmed influenza infection, from January 2012December 2018, at a tertiary care hospital. The overall mortality rate in our study was found to be $13.4 \%$, which is comparable to similar studies done globally and also from other countries in region [12]. The ICU stay, along with the presence of septic shock and acute renal failure, were found to be strong predictors of mortality in our hospitalised patients with influenza. In this study, $20.5 \%$ of the influenza patients received treatment in the ICU which is found to be higher than the number reported in the literature $[13,14]$. As our hospital's ICU only admits patients requiring invasive mechanical ventilation and level 3 care, the mortality rates are therefore indicative of severity of the disease, leading to ARDS and the need of mechanical ventilation. The ICU mortality rate in this study was found to be $47.8 \%$ which is higher than other ICU mortality rates observed worldwide [14-17]. Our ICU patients were older with average age of 66 , with diabetes and hypertension in $2 / 3^{\text {rd }}$ of them, which might be contributing, to high mortality. This indicates that, severe form of influenza is associated with high mortality, compared to milder form of the disease. Septic shock in our study was found to be a very strong predictor of mortality in influenza patients, being present in 11 out of 15 patients $(73.33 \%)$ who did not survive compared to only $6 \%$ of survivors. Difficult management of septic shock, in the presence of worsening hypoxemia and ARDS seems to be the likely reason behind these results [18]. This association between septic shock and influenza, though was not directly compared in previous studies done globally but reported in literature $[19,20,22]$.

In our study $10 / 15$ that is $66.66 \%$ of non-survivors had renal failure. This appeared to be another strong predictor of influenza mortality, as is also shown, in most of the studies done globally [22-24]. The mortality rate was higher in middle-aged group, in spite of the lower incidence in current study. This is consistent with results of the previous studies, which also showed higher mortality among middle aged group [25,26]. We need further large-scale investigations to identify the true relationship between age group and influenza mortality In our study, females had lower mortality rates compared to males, even though the majority of the influenza patients were females. Although previous literature regarding influenza related complications showed higher mortality in male patients as compared to female, it would be inappropriate to assume association of male gender with increased risk of mortality due to non-availability of sufficient epidemiological data [27,28]. Time from onset of symptoms to hospital admission was found to be associated with increased risk of mortality in our study. Delay in seeking medical care with severe influenza, hence resulting in late initiation of antiviral therapy are associated with poor outcomes [29,30]. This is also signifying a lack of primary and secondary care facilities in our country, where patients are instead brought to tertiary care facilities, with more severe and advance stage of disease. In present study, only 17 patients had positive sputum culture for secondary bacterial or fungal infection. This could be attributed to early initiation of empirical antibiotics at our institute. Staphylococcus Aureus, as expected, was the commonest organism isolated, which is in line with all previous reports $[31,32]$. Similarly, fungal infections were also found to be the second highest reported infection, in our study. Influenzas infection is a risk factor for invasive pulmonary aspergillosis even in immunocompetent patients and can lead to invasive disease [33,34]. The pathogen Aspergillus fumigatus was isolated in 5 out of 17 culture positive patients. All patents with Aspergillus isolated in respiratory secretion had underlying obstructive airway diseases. This finding can therefore be due to colonization of respiratory tract by the organism, as all patients improved without any antifungal therapy. The high incidence of co-infections might be result of some immune defect in these patients, making them more susceptible to acquire these infections.

Our study has few limitations. It is a single centre study done at a tertiary care university hospital. The number of cases is therefore limited, and they are also more severe in intensity as compared to cases in district hospitals. The findings observed from this university hospital study might not be generalizable to other hospitals. The lack of disease severity scoring system like APACHE or SOFA makes it difficult to truly ascertain the risk of mortality in these patients. Being a retrospective study, we might have some inadequate data. Our sample size was small, and the results observed might change if the study is done with a larger sample size.

Despite the above limitations, our study has shown novel findings about mortality prediction in hospitalized influenza patients during five-years period. The mortality of influenza in our institute was $13 \%$ but it reached a significantly high percentage of $47.2 \%$ in severe influenza patient requiring mechanical ventilation and ICU admissions. 
Table 1. Predictors of mortality in influenza.

\begin{tabular}{|c|c|c|c|}
\hline & Survivors (n=97) & Died $(n=15)$ & p \\
\hline Age (years) & $57.9 \pm 17.6$ & $59.5 \pm 17.6$ & 0.72 \\
\hline Gende & & & 0.17 \\
\hline Male & $34(35.1)$ & $8(53.3)$ & \\
\hline Female & $63(64.9)$ & $7(46.7)$ & \\
\hline Body mass index & $27.3 \pm 9.0$ & $25.0 \pm 2.9$ & 0.35 \\
\hline Duration, illness-admission & $5.1 \pm 2.7$ & $7.0 \pm 5.2$ & $0.03^{*}$ \\
\hline Length of ICU stay & $7.7 \pm 5.8$ & $10.2 \pm 6.6$ & $0.33^{*}$ \\
\hline Length of stay & $6.5 \pm 6.1$ & $12.2 \pm 7.8$ & $0.01^{*}$ \\
\hline $\begin{array}{l}\text { Comorbidities } \\
\text { Diabetes mellitus } \\
\text { Hypertension } \\
\text { Asthma } \\
\text { Chronic lung disease } \\
\text { Heart disease } \\
\text { Kidney disease } \\
\text { Liver disease } \\
\text { Neurology disease } \\
\text { Immunosuppression } \\
\text { Blood disease } \\
\text { Pregnancy }\end{array}$ & $\begin{array}{l}45(46.4) \\
46(47.4) \\
12(12.4) \\
15(15.5) \\
14(14.4) \\
11(11.3) \\
3(3.1) \\
4(4.1) \\
6(6.2) \\
1(1.0) \\
13(13.4)\end{array}$ & $\begin{array}{c}6(40) \\
8(53.3) \\
2(13.3) \\
3(20) \\
4(26.7) \\
1(6.7) \\
0 \\
0 \\
0 \\
2(13.3) \\
1(6.7)\end{array}$ & $\begin{array}{l}0.64 \\
0.67 \\
0.91 \\
0.65 \\
0.23 \\
0.58 \\
0.49 \\
0.42 \\
0.32 \\
0.04 \\
0.68\end{array}$ \\
\hline $\begin{array}{l}\text { Symptoms } \\
\text { Fever } \\
\text { Cough } \\
\text { Sputum } \\
\text { Myalgia } \\
\text { Headache } \\
\text { Diarrhoea } \\
\text { Nausea and vomiting } \\
\text { Confusion } \\
\text { Dyspnoea } \\
\text { Hypotension } \\
\text { Muscle weakness } \\
\text { General weakness } \\
\text { Upper respiratory tract infection symptoms } \\
\text { Arrhythmia }\end{array}$ & $\begin{array}{c}90(92.8) \\
87(89.7) \\
38(39.2) \\
14(14.4) \\
2(2.1) \\
7(7.2) \\
8(8.2) \\
1(1.0) \\
58(59.8) \\
5(5.2) \\
6(6.2) \\
8(8.2) \\
29(29.9) \\
7(7.2)\end{array}$ & $\begin{array}{c}14(93.3) \\
14(93.3) \\
5(33.3) \\
3(20.0) \\
0 \\
3(20) \\
1(6.7) \\
1(6.7) \\
10(66.7) \\
2(13.3) \\
0 \\
0 \\
4(26.7) \\
6(40.0)\end{array}$ & $\begin{array}{l}0.93 \\
0.99 \\
0.66 \\
0.69 \\
0.99 \\
0.13 \\
0.99 \\
0.25 \\
0.61 \\
0.23 \\
0.99 \\
0.59 \\
0.99 \\
0.002\end{array}$ \\
\hline $\begin{array}{l}\text { Influenza type } \\
\text { A } \\
\text { B }\end{array}$ & $\begin{array}{l}73(75.3) \\
24(24.7)\end{array}$ & $\begin{array}{c}14(93.3) \\
1(6.7)\end{array}$ & 0.18 \\
\hline ICU stay & $12(12.4)$ & $11(73.3)$ & $<0.001$ \\
\hline Ventilation & $10(10.3)$ & $10(66.7)$ & $<0.001$ \\
\hline Procalcitonin & $4.7 \pm 13.5$ & $12.6 \pm 13.5$ & $0.04^{*}$ \\
\hline $\begin{array}{l}\text { Treatment } \\
\text { Steroid }\end{array}$ & $51(52.6)$ & $10(66.7)$ & 0.30 \\
\hline TA/sputum positive & $14(17.7)$ & $3(27.3)$ & 0.42 \\
\hline Bacterial pneumonia & $56(57.7)$ & $11(73.3)$ & 0.25 \\
\hline Exacerbation of underlying disease & $14(14.4)$ & $1(6.7)$ & 0.68 \\
\hline Respiratory failure & $19(19.6)$ & $8(53.3)$ & 0.008 \\
\hline Acute respiratory distress syndrome & $9(9.3)$ & $7(46.7)$ & 0.001 \\
\hline Pneumothorax & $3(3.1)$ & 0 & 0.99 \\
\hline Pulmonary disease & $13(13.4)$ & $1(6.7)$ & 0.68 \\
\hline Acute coronary syndrome & $11(11.3)$ & $5(33.3)$ & 0.03 \\
\hline Arrhythmias & $7(7.2)$ & $1(6.7)$ & 0.99 \\
\hline Sepsis/septic shock & $6(6.2)$ & $11(73.3)$ & $<0.001$ \\
\hline Acute kidney injury & $23(24)$ & $11(73.3)$ & $<0.001$ \\
\hline Cerebrovascular accident & $2(2.1)$ & 0 & 0.99 \\
\hline Seizure & $1(1.0)$ & 0 & 0.99 \\
\hline
\end{tabular}


Table 2. Factors predicting poor outcome.

\begin{tabular}{lcc} 
& Odd ratio $(95 \% \mathrm{CI})$ & $\mathrm{p}$ \\
Sepsis/septic shock & & \\
No & 1.0 & $<0.001$ \\
Yes & $45.24(6.20-330)$ & \\
Acute kidney injury & 1.0 & \\
$\quad$ No & $10.88(1.61-73.52)$ & 0.01 \\
Yes & 1.0 & \\
\hline ICU stay & & \\
No & $17.22(2.68-110.54)$ & 0.003 \\
Yes &
\end{tabular}

\section{Conclusions}

Our study has showed that development of concomitant septic shock and renal failure increases the odds of mortality in influenza patients. This finding might help clinicians in more vigilant care of these patients with earlier ICU transfers and probable use of invasive hemodynamic monitoring devices in management of these patients.

\section{References}

1. Blut A. Influenza virus. Transfus Med Hemother 2009;36:32-9.

2. Caini S, Kroneman M, Wiegers T, et al. Clinical characteristics and severity of influenza infections by virus type, subtype, and lineage: A systematic literature review. Influenza Other Respir Viruses 2018;12:780-92.

3. Fischer WA 2nd, Gong M, Bhagwanjee S, Sevransky J. Global burden of influenza as a cause of cardiopulmonary morbidity and mortality. Glob Heart 2014;9:325-36.

4. Iuliano AD, Roguski KM, Chang HH, et al. Estimates of global seasonal influenza-associated respiratory mortality: a modelling study. Lancet 2018;391:1285-300. Erratum in: Lancet 2018;391:1262.

5. Nielsen J, Vestergaard LS, Richter L, et al. European all-cause excess and influenza-attributable mortality in the 2017/18 season: should the burden of influenza B be reconsidered? Clin Microbiol Infect 2019;25:1266-76.

6. El Guerche-Séblain C, Caini S, Paget J, et al. Epidemiology and timing of seasonal influenza epidemics in the Asia-Pacific region, 2010-2017: implications for influenza vaccination programs. BMC Public Health 2019;19:331.

7. Murhekar M, Mehendale S. The 2015 influenza A (H1N1) pdm09 outbreak in India. Indian J Med Res 2016;143:821-3.

8. Bashir F, Fawad Khan K, Zafar Qureshi S, et al. Laboratory based influenza sentinel surveillance in Pakistan at NIC, Islamabad 2007-2017. Eur J Public Health 2019;29:ckz186.499.

9. Troeger CE, Blacker BF, Khalil IA, et al. Mortality, morbidity, and hospitalisations due to influenza lower respiratory tract infections, 2017: an analysis for the Global Burden of Disease Study 2017. Lancet Respir Med 2019;7:69-89.

10. Kshatriya R, Khara N, Ganjiwale J, et al. Lessons learnt from the Indian H1N1 (swine flu) epidemic: Predictors of outcome based on epidemiological and clinical profile. J Family Med Prim Care 2018;7:1506-9.

11. Mertz D, Kim TH, Johnstone J, et al. Populations at risk for severe or complicated influenza illness: systematic review and meta-analysis. BMJ 2013;347:f5061.

12. Paget J, Spreeuwenberg P, Charu V, et al. Global mortality associated with seasonal influenza epidemics: New burden estimates and predictors from the GLaMOR Project. J Glob Health 2019;9:020421.

13. Chow EJ, Doyle JD, Uyeki TM. Influenza virus-related critical illness: prevention, diagnosis, treatment. Critical Care 2019;23:214

14. Adlhoch C, Gomes Dias J, Bonmarin I, et al. Determinants of fatal outcome in patients admitted to intensive care units with influenza, European Union 2009-2017. Open Forum Infect Dis 2019;6:ofz462.

15. Beumer M, Koch R, van Beuningen D, et al. Influenza virus and factors that are associated with ICU admission, pulmonary coinfections and ICU mortality. J Critical Care 2019;50:59-65.

16. Taylor G, Abdesselam K, Pelude L, et al. Epidemiological features of influenza in Canadian adult intensive care unit patients. Epidemiol Infect 2016;144:741-50.

17. Chao CM, Lai CC, Chan KS, et al. Outcomes of patients with severe influenza infection admitted to intensive care units: a retrospective study in a medical center. J Med Microbiol 2017;66:1421-8.

18. Florescu DF, Kalil AC. The complex link between influenza and severe sepsis. Virulence 2014;5:137-42.

19. Shi T, Nie Z, Huang L, et al. Mortality risk factors in children with severe influenza virus infection admitted to the pediatric intensive care unit. Med (Baltimore). 2019;98:e16861.

20. Martínez A, Soldevila N, Romero-Tamarit A, et al. Risk factors associated with severe outcomes in adult hospitalized patients according to influenza type and subtype. PloS One 2019;14:e0210353.

21. Abdulkader RC, Ho YL, de Sousa Santos S, et al. Characteristics of acute kidney injury in patients infected with the 2009 influenza A (H1N1) virus. Clin J Am Soc Nephrol 2010;5:1916-21.

22. Ho YC, Wang JL, Wang JT, et al. Prognostic factors for fatal adult influenza pneumonia. J Infect 2009;58:439-45.

23. Martin-Loeches I, Papiol E, Rodriguez A, et al. Acute kidney injury in critical ill patients affected by influenza A (H1N1) virus infection. Critical care 2011;15:R66.

24. Watanabe T. Renal complications of seasonal and pandemic influenza A virus infections. Eur J Pediatr 2013;172:15-22.

25. Borse R, Kadam D, Sangle S, et al. Clinicoradiologic correlation in adult patients diagnosed with novel influenza A (H1N1). J Assoc Physicians India 2013;61:600-7.

26. Chowell G, Bertozzi SM, Colchero MA, et al. Severe respiratory disease concurrent with the circulation of H1N1 influenza. New Eng J Med 2009;361:674-9.

27. Huang WT, Chang CH, Hsu YF, Chuang JH. Prognostic factors for mortality in patients hospitalized with influenza complications, in Taiwan. International health 2015;7:73-5.

28. Kadel S, Kovats S. Sex hormones regulate innate immune cells and promote sex differences in respiratory virus infection. Front Immunol 2018;9:1653.

29. Chowell G, Viboud C, Simonsen L, et al. Impact of antiviral treatment and hospital admission delay on risk of death associated with $2009 \mathrm{~A} / \mathrm{H} 1 \mathrm{~N} 1$ pandemic influenza in Mexico. BMC Infect Dis 2012;12:97.

30. Kumar A. Early versus late oseltamivir treatment in severely ill patients with 2009 pandemic influenza A (H1N1): speed is life. J Antimicrob Chemother 2011;66:959-63.

31. Borgogna TR, Hisey B, Heitmann E, et al. Secondary bacterial 
pneumonia by Staphylococcus aureus following influenza A infection is SaeR/S dependent. J Infect Dis 2018;218:809-13.

32. Klein EY, Monteforte B, Gupta A, et al. The frequency of influenza and bacterial co infection: a systematic review and meta-analysis. Influenza Other Respir Viruses 2016;10:394-403.

33. Schauwvlieghe AF, Rijnders BJ, Philips N, et al. Invasive aspergillosis in patients admitted to the intensive care unit with severe influenza: a retrospective cohort study. Lancet Respir Med 2018;6:782-92.

34. Crum-Cianflone NF. Invasive aspergillosis associated with severe influenza infections. Open Forum Infect Dis 2016;3:ofw171. 